\title{
Beyond Mortality: Violent Injury Surveillance Using NC DETECT ED Visit Data
}

\author{
Katherine J. Harmon*1, Amy Ising ${ }^{1}$, Scott Proescholdbell ${ }^{2}$ and Anna Waller ${ }^{1}$ \\ 'University of North Carolina at Chapel Hill, Chapel Hill, NC, USA; 'Injury and Violence Prevention Branch, NC Division of Public \\ Health, NC Department of Health and Human Services, Raleigh, NC, USA
}

\section{Objective}

To describe violent injuries treated in North Carolina (NC) emergency departments (EDs) and compare to deaths reported by the NC Violent Death Reporting System (NC-VDRS).

\section{Introduction}

Violence-related injuries are a major source of morbidity and mortality in NC. From 2005-2014, suicide and homicide ranked as NC's $11^{\text {th }}$ and $16^{\text {th }}$ causes of death, respectively. In 2014, there were 1,932 total violent deaths, of which 1,303 were due to suicide (67\%), 536 due to homicide (28\%), and 93 due to another mechanism of violent injury (5\%). These deaths represent a fraction of the total number of violence-related injuries in NC. ${ }^{1}$ This study examined ED visit data captured by NC DETECT to identify and describe violent injuries treated in NC EDs and compare/contrast with fatalities reported by NC-VDRS.

\section{Methods}

This descriptive epidemiologic study included all NC ED visits made to a $24 / 7$, acute-care, civilian, hospital-affiliated ED from January 1, 2012 - September 30, 2015 reported to NC DETECT with an injury mechanism code indicating a violent injury due to one of the following injury types: self-harm, assault, legal intervention, or unintentional firearm. In addition, ED visits of an undetermined intent were separately examined. Violence-related NC ED visits were classified according to definitions developed by the National Center for Injury Prevention and Control for WISQARS ${ }^{\mathrm{TM}} .{ }^{2}$ Descriptive analyses consisted of counts, percentages, and incidence rates.

\section{Results}

From January 1, 2012-September 30, 2015, there were 182,385 violence-related NC ED visits captured by NC DETECT (492.1/100,000 person-years). The most common type of violent injury treated in NC EDs was assault, with 132,550 visits (357.6/100,000 person-years), followed by self-inflicted injury (41,455 visits; 111.8/100,000 person-years), unintentional firearmrelated injury (5,940 visits; $15.9 / 100,000$ person-years), and legal intervention (2,440 visits; 6.6/100,000 person-years). Twelve percent of all violence-related NC ED visits were made by children $0-17$ years of age (21,876 ED visits). There were an additional 20,867 NC ED visits for injuries of an undetermined intent.

Males visited a NC ED for treatment of violent injuries more often than females (550.3 versus 436.2 visits/100,000 person-years, respectively). Young adults 20-24 years of age had the highest rate of violence-related NC ED visits $(1,242.9)$, followed by individuals aged 25-34 (997.4), 15-19 (935.3), 35-44 (635.6) and 45-54 (461.3) (visits/100,000 person-years in parentheses). Among violence-related NC ED visits, the most common mechanism of injury was struck by/against an object or person $35.0 \%$ ), the most common mode of transport to the ED was private transportation $(37.7 \%)$, the most common discharge disposition was discharged home from the ED $(77.7 \%)$, the most common expected source of payment was self-pay $(37.5 \%)$, and the most common time of visit was the evening hours of 6-11 PM (33.6\%).

Violence-related ED visits differed from violence-related deaths reported by the NC-VDRS. On average, there were 25 times more annual violence-related ED visits than deaths. Table 1 displays the average annual number of violence-related ED visits and deaths stratified by type of violent injury. The proportion of ED visits due to assault was greater than the proportion of deaths due to homicide, while the proportion of injuries/deaths attributable to self-inflicted injury/ suicide was higher among fatalities. A comparison of the self-inflicted injury and suicide data shows that women have a higher rate of ED visits for self-harm while men have a higher rate of suicide (data not shown).

\section{Conclusions}

Violence-related injuries are a common source of morbidity and mortality in NC. The annual number of violence-related ED visits exceeds the number of violence-related deaths, 25 to 1 . Because there are important differences between violent injury leading to ED visits and fatalities, comparing NC DETECT surveillance with NC-VDRS fatality data expands our understanding of violent injury in $\mathrm{NC}$ and better informs prevention efforts.

Table 1. Violence-Related North Carolina Emergency Department Visits and Deaths

\begin{tabular}{|c|c|c|}
\hline Type of Violent Injury & $\begin{array}{c}\text { ED Visits } \\
\text { Average Annual Number } \\
2012-2015 \\
\text { Number }(\%)\end{array}$ & $\begin{array}{c}\text { Deaths } \\
2014 \\
\text { Number (\%) }\end{array}$ \\
\hline Assault/Homicide & $35,347(72.7 \%)$ & $536(28.5 \%)$ \\
\hline Self-Inflicted/Suicide & $11,055(22.7 \%)$ & $1,303(69.3 \%)$ \\
\hline Unintentional Firearm & $1,584(3.3 \%)$ & $16(0.9)$ \\
\hline Legal Intervention & $651(1.3 \%)$ & $25(1.3 \%)$ \\
\hline Total & $48,636(100.0 \%)$ & $1,880(100.0 \%)$ \\
\hline
\end{tabular}

Average annual estimates were calculated from NC DETECT ED visit data from Jan. 1, 2012 - Sept. 30, 2015.

\section{Keywords}

Violence; Injury; Surveillance; Morbidity; Emergency Department

\section{Acknowledgments}

NC DETECT is a statewide public health syndromic surveillance system, funded by the NC Division of Public Health (NC DPH) Federal Public Health Emergency Preparedness Grant and managed through collaboration between NC DPH and UNC-CH Department of Emergency Medicine's Carolina Center for Health Informatics. The NC DETECT Data Oversight Committee does not take responsibility for the scientific validity or accuracy of methodology, results, statistical analyses, or conclusions presented.

\section{References}

1. Injury and Violence Prevention Branch (IVPB). North Carolina Violent Death Reporting System Annual Report 2014. Raleigh: NC. IVPB, NC DPH, NC DHHS, 2017. Available at: www.injuryfreenc.ncdhhs.gov/ DataSurveillance/VDRS/2014-NC-VDRS-AnnualReport-Final.pdf.

2. National Center for Injury Prevention and Control (NCIPC). Definitions of Web-based Injury Statistics Query and Reporting System (WISQARSTM) Nonfatal website. NCIPC, CDC. www.cdc.gov/ ncipc/wisqars/nonfatal/definitions.htm. Last updated March 21, 2007. Accessed June 21, 2017

\section{${ }^{*}$ Katherine J. Harmon}

E-mail: kjharmon@email.unc.edu 